\title{
Video Article \\ A Postoperative Evaluation Guideline for Computer-Assisted Reconstruction of the Mandible
}

\author{
Gustaaf J. C. van Baar ${ }^{1}$, Niels P. T. J. Liberton ${ }^{2}$, Henri A. H. Winters ${ }^{3}$, Lars Leeuwrik ${ }^{1}$, Tymour Forouzanfar ${ }^{1}$, Frank K. J. Leusink ${ }^{1}$ \\ ${ }^{1}$ Department of Oral and Maxillofacial Surgery/Pathology, Amsterdam UMC and Academic Centre for Dentistry Amsterdam (ACTA), Vrije Universiteit Amsterdam \\ ${ }^{2}$ Medical Technology, 3D Innovation Lab, Amsterdam UMC, Vrije Universiteit Amsterdam \\ ${ }^{3}$ Department of Plastic, Reconstructive and Hand Surgery, Amsterdam UMC, Vrije Universiteit Amsterdam
}

Correspondence to: Gustaaf J. C. van Baar at g.vanbaar@amsterdamumc.nl

URL: https://www.jove.com/video/60363

DOI: doi:10.3791/60363

Keywords: Medicine, Issue 155, oral cancer, mandibular reconstruction, free tissue flaps, surgery computer-assisted, computer-aided design, computer-aided manufacturing, data accuracy, software

Date Published: 1/28/2020

Citation: van Baar, G.J.C., Liberton, N.P.T.J., Winters, H.A.H., Leeuwrik, L., Forouzanfar, T., Leusink, F.K.J. A Postoperative Evaluation Guideline for Computer-Assisted Reconstruction of the Mandible. J. Vis. Exp. (155), e60363, doi:10.3791/60363 (2020).

\section{Abstract}

Valid comparisons of postoperative accuracy results in computer-assisted reconstruction of the mandible are difficult due to heterogeneity in imaging modalities, mandibular defect classification, and evaluation methodologies between studies. This guideline uses a step-by-step approach guiding the process of imaging, classification of mandibular defects and volume assessment of three-dimensional (3D) models, after which a legitimized quantitative accuracy evaluation method can be performed between the postoperative clinical situation and the preoperative virtual plan. The condyles and the vertical and horizontal corners of the mandible are used as bony landmarks to define virtual lines in the computer-assisted surgery (CAS) software. Between these lines the axial, coronal, and both sagittal mandibular angles are calculated on both pre- and postoperative 3D models of the (neo)mandible and subsequently the deviations are calculated. By superimposing the postoperative $3 \mathrm{D}$ model to the preoperative virtually planned 3D model, which is fixed to the XYZ axis, the deviation between pre- and postoperative virtually planned dental implant positions can be calculated. This protocol continues and specifies an earlier publication of this evaluation guideline.

\section{Video Link}

The video component of this article can be found at https://www.jove.com/video/60363/

\section{Introduction}

Computer-assisted surgery (CAS) in reconstructive surgery involves four consecutive phases: a virtual planning phase, a three-dimensional (3D) modeling phase, a surgical phase, and a postoperative evaluation phase ${ }^{1}$. The planning phase starts with the obtainment of a craniofacial computed tomography (CT) scan, and a donor site CT or CT angiography (CTA) scan. Diverse tissue types correspond to an amount of Xray attenuation, which leads to scan voxels with a specific gray value ranged according to Hounsfield units (HU) (human bone [+1000 HU], water [0 HU], and air [-1000 HU]). These images are stored in Digital Imaging and Communications in Medicine (DICOM) file format. By selecting the regions of interest (ROls) in segmentation software, 3D models can be generated ${ }^{2}$. The most popular and feasible segmentation technique is thresholding: voxels above a selected $\mathrm{HU}$ threshold value are enclosed in the ROI. These voxels are subsequently converted into 3D models in the Standard Tessellation Language (STL) file format ${ }^{3}$, and uploaded into CAS software to plan the osteotomies and to design 3D devices ${ }^{4}$. During the modeling phase, the designed devices are 3D printed and sterilized, followed by the surgical phase. The final evaluation phase consists of a postoperative CT scan of the patient's skull, followed by an accuracy analysis comparing the postoperative result with the preoperative virtual plan.

Our recently published systematic review regarding accuracy of computer-assisted mandibular reconstructions showed heterogeneity in image acquisition, classification of mandibular defects, and evaluation methodologies. This heterogeneity limits valid comparisons of postoperative hard tissue accuracy results between studies ${ }^{5}$. Standardization of CAS phases in the process of mandibular reconstruction is important due to the new European Union medical device regulation (MDR), which demands Conformité Européenne (CE) certification for all different CAS processes, and which will be operational from spring $2020^{6}$. Here, we present a practical, feasible and reproducible evaluation guideline for computer-assisted reconstructions of the mandible in order to create uniformity between studies regarding postoperative accuracy evaluation. This protocol continues and specifies an earlier publication of this evaluation guideline ${ }^{7}$, which is currently being tested in a large multicenter cohort study in which all different types of mandibular reconstructions will be analyzed for their accuracy aiming to discover tolerable outcome ranges regarding functionality. 


\section{Protocol}

The Medical Ethics Review Committee of VU University Medical Center (registered with the US Office for Human Research Protections [OHRP] as IRB00002991) confirmed that the Medical Research Involving Human Subjects Act (WMO) does not apply to this study. The FWA number assigned to VU University Medical Center is FWA00017598.

NOTE: Validate all steps in this protocol independently by two different observers.

\section{Skull and donor site imaging}

1. Execute both pre- and postoperative scanning with a multiple detector $\mathrm{CT}$ (MDCT), using the same machine and scanner settings, with the parameter slice thickness (ST) set $<1.25 \mathrm{~mm}$. Perform the postoperative MDCT scan within six weeks after reconstruction. NOTE: In case of adjuvant radiation therapy, use the first postoperative MDCT scan prior to the therapy.

\section{Classification of the mandibular defect}

1. Classify the mandibular defect according to the classification of Brown et al. ${ }^{8}$.

\section{Segmentation of the DICOM images of the postoperative CT scan}

1. Open the image-based 3D medical software (e.g., Mimics inPrint 3.0). Click File and New from Disk, then a folder window will open. Select the folder that contains the DICOM images of the postoperative CT scan to import (select the whole folder), choose the right study in the list and click Convert. A window will pop up for assessment of the orientation of the skull.

2. Change the orientation by left clicking the orientation characters; click OK to validate.

3. Perform the 5-step segmentation workflow.

1. To create ROI, click the Thresholding tool. Create the ROI by defining a threshold containing all the voxels of the mandibular bone within a certain interval of gray values, which is proportional to the density of the bony tissue. Manually tweak the Hounsfield range by moving the two sliders on the left and the right. Click the green button to validate the segmentation.

NOTE: The thresholding tool allows the user to select the bone within a range of density, expressed in Hounsfield Units. After this step, a new ROI appears in the ROI tab and the software jumps to the second step of the workflow.

2. To edit ROI, choose the Isolate tool; click the mandible in the 3D viewport, which will be automatically isolated from the cranium and becomes green. Select the option Create result in new ROI. Click the green button to validate the isolation and subsequently all the non-connected structures disappear. Rename the ROI ("Mandible Post-op").

NOTE: Optionally, use the Lasso tool to remove scattering by editing the ROI directly on the images or in the 3D viewport. When the CT scan is of poor quality, the condyles can be connected to the skull. In that case, click the Split tool, which asks the user to define a foreground and a background. Select Foreground and select the mandible thorough the axial or coronal coupes. Select Background and select the maxilla and cranium thorough the axial or coronal coupes. The region corresponding with the foreground will be kept in the $\mathrm{ROI}$ and the region corresponding with the background will be deleted. Click the green button to validate.

3. When ROI is finished and ready to be converted into a 3D model, click the Add Part button of the workflow toolbar. Click the Solid Part tool. Select the solid part Mandible Post-op and select Off in the Smoothing options. Click the green button to validate.

4. When the parts are constructed, the software automatically goes to the fourth step of the workflow: edit part. With the contours of the created parts shown on the images, assess the accuracy of the parts. Skip the Smooth tool.

5. In the last step of the workflow (prepare print), select the Mandible Post-op part in the export menu, choose the output directory, select the 1,00 scale and click the green button to validate.

NOTE: The "Mandible Post-op" part is now exported as an .STL file.

\section{4. $\mathrm{XYZ}$ axis orientation}

NOTE: The preoperative STL model includes the cranium, (neo)mandible, and the virtually planned dental implants (if planned). Note that the evaluation works easier with separated STL files of the cranium and skull, but still in fixed position to each other. When the preoperative STL model of the cranium and the mandible are merged, use the 3D medical software (following the steps described above) to split the mandible from the cranium.

1. Open the evaluation software (Table of Materials). Drag the preoperative STL file (including virtual plan) in the popped-up screen.

2. Determine the Frankfurt plane, midsagittal plane, and the nasion for uniform orientation of the preoperative STL model of the skull on the XYZ axis.

1. Click Construct | Plane | 3-Point Plane and create a virtual point by using Ctrl + left click both the internal acoustic foramina and the left infraorbital margin (Frankfurt plane) ${ }^{9}$. Click Create and close after pointing on the STL model.

2. Click Construct | Line | 2-Point Line and create a virtual point by using Ctrl + left click the nasion and the basion (midsagittal plane) ${ }^{10}$.

3. Click Construct | Point | Point and create a virtual point by using Ctrl + left click the nasion.

4. Click Operations | Main Alignment | Plane-Line-Point. Combine the actual parameter "Plane 1" with the nominal parameter "Plane Z", the actual parameter "Line 1" with the nominal parameter "Line Y", and the actual parameter "Point 1" with the nominal parameter "Global coordinate system". 
NOTE: The preoperative STL models of the cranium and (neo)mandible are now fixed to the XYZ axis (Figure 1).

\section{Volume assessment of the pre- and postoperative STL models}

NOTE: Examine pre- and postoperative STL models on volume similarity to rule out volume inaccuracies between the two models as much as possible, since they can influence accuracy measurements.

1. Select the STL file of only the preoperative (neo)mandible under Actual Elements, where all "Meshes" are shown. Click Operations | CAD | Actual Mesh To CAD. Select New CAD data in the popped-up menu, rename the file (e.g., "Mandible Pre-op") and click OK. NOTE: The preoperative STL model is now visible under Nominal Elements | CAD in the left explorer menu.

2. Drag the postoperative STL model into the software (created during section 3 of the protocol). Rename the file (e.g., "Mandible Post-op"). Select the STL file under Actual Elements in the left explorer menu where all "Meshes" are shown. Click Operations | Alignment | Single Element Transformation | 3-Point Alignment.

3. In the popped-up menu, combine 3 "Nominal points" on the "Mandible Pre-op" (e.g., condyle superior, horizontal, and vertical corner of the mandible) with 3 similar "Actual points" on the "Mandible Post-op" by ctrl + left clicking. Validate with Apply and Close.

NOTE: The STL models will be roughly superimposed on each other based on these 3 landmarks. This will speed up the calculations of the software during the next steps.

4. Deselect the Mandible Pre-op and select the Mandible Post-op in the left explorer menu. Click the Select/Deselect on Surface tool in the bottom toolbar. Select a surface on the remnant mandible on both the lateral and medial sides (not in touch with the osteosynthesis material).

5. Click Operations | Alignment | Main Alignment | Local Best Fit. Select All CAD groups as the targeted element in the popped-up menu. Take a maximum distance of $10.000 \mathrm{~mm}$. Validate with Apply and Close.

NOTE: The selected part of the remnant mandible of the "Mandible Post-op" will be accurately superimposed on the similar part of the "Mandible Pre-op". Now both models are ready for the STL volume assessment.

6. Click the Select/Deselect on Surface tool in the bottom toolbar. Select a surface on only the lateral side within the surface of the previous step. Click Inspection I CAD Comparison | Surface Comparison on Actual. Use a maximum distance of $10.00 \mathrm{~mm}$ in the popped-up menu and validate with OK.

7. Turn off the Toggle Visibility of the Mandible Post-op. Use the Select Patch tool, left-click the selected surface. Click the magnifying glass on the toolbar above. A round toolbar pops up in the screen. Click Check | Deviation Label Arithmetic Mean and the arithmetic mean in $\mathrm{mm}$ will be shown (Figure 2).

8. In case of an arithmetic mean $<0.5 \mathrm{~mm}$, continue to section 6 of this protocol. In case of an arithmetic mean $>0.5 \mathrm{~mm}$, repeat the postoperative CT scan (DICOM file) segmentation in the 3D medical software by adjusting the threshold values. Repeat the segmentation and superimposition until an arithmetic mean $<0.5 \mathrm{~mm}$ is achieved.

NOTE: The two STL volumes are now ready for valid accuracy comparisons.

\section{Superimposition of the condylar processes}

1. Deselect the Mandible Pre-op and select the Mandible Post-op in the left explorer menu. Click the Select/Deselect on Surface tool in the bottom toolbar. Select the whole surfaces of both condyles by drawing planes (lateral and medial side) from the most caudal point of the incisura mandibulae (mandibular notch) perpendicular to the posterior edge of the border between condyle and the vertical corner.

2. Click Operations | Alignment | Main Alignment | Local Best Fit. Select All CAD groups as the targeted element in the popped-up menu. Take a maximum distance of $10.000 \mathrm{~mm}$. Validate with Apply and Close.

NOTE: The selected condyles of the "Mandible Post-op" will be accurately superimposed on condyles of the "Mandible Pre-op" (Figure 3).

\section{Calculation of the coronal, axial, and sagittal mandibular angles}

NOTE: The identification of bony landmarks is performed separately on the "Mandible Pre-op" and "Mandible Post-op" STL models. Deselect the Mandible Post-op while identifying bony landmarks in the "Mandible Pre-op", and vice versa.

1. Select the Mandible Pre-op in the left explorer menu. Click Construct | Point | Surface Point to determine virtual points on the condyle superior (CS), condyle posterior (CP), vertical corner (VC), and horizontal corner (HC) according to the classification of Brown et al. ${ }^{8}$.

2. Select the Mandible Post-op in the left explorer menu. Click Construct | Point | Projection Point to determine virtual points on the CS, CP, $\mathrm{VC}$, and $\mathrm{HC}$ according to the classification of Brown et al. ${ }^{8}$.

NOTE: For Brown class Ic, Ilc or IVc defects, determine virtual points on the most superior and posterior part of the vertical segment of bone graft or the titanium/prosthetic condyle. If the mandibular resection includes one or more corners, select the most inferior point of the osteotomy plane between the two segments of bone graft. When the mandibular resection includes only half of a horizontal or vertical corner (remnant mandible next to a segment of bone graft), determine a virtual point on the segment of bone graft on the most inferior part of the osteotomy plane. In case of a Brown class I mandibular defect, determine a virtual point on the most anterior and inferior part of the horizontal segment of bone graft and consider this virtual point as the horizontal corner. In case of (extra) osteotomies outside the anatomical vertical or horizontal corner, determine the osteotomy closest to these corners as vertical or horizontal corner.

3. To create a line between 2 virtual points, click Construct | Line | 2-Point Line. Select 2 points under construction elements in the poppedup menu to connect them with a line. Click Create and close.

4. Create a midsagittal plane in both the Mandible Pre-op and Mandible Post-op by clicking Construct | Plane | Plane in Viewing Directions. Select 2 points on the Z-axis.

5. To create an angle between 2 lines, or between a line and a plane, click Construct | Angle | 2-Directions Angle. Subsequently select Line $\mathbf{1}$, and Line/Plane 2 in the popped-up menu. Click Create and close.

6. Connect all the Mandible Pre-op angles to the Mandible Post-op angles by selecting a Mandible Pre-op angle in the explorer menu, then click Magnifying Glass | Measuring Principle | Link to Actual Element. Select the corresponding Mandible Post-op angle, and click OK. 
7. With this knowledge, determine the right and left coronal mandibular angles between the lines from CS to VC and the midsagittal line (ML).

1. Determine the right and left axial mandibular angles between the lines from VC to HC and ML.

2. Determine the sagittal mandibular angles between the lines from $\mathrm{CP}$ to $\mathrm{VC}$ and the lines from VC to HC.

3. Calculate and report the deviations in degrees $\left({ }^{\circ}\right)$ between the postoperative angles and the virtual planned angles.

\section{Calculation of the $X Y Z$ deviations and distance $X Y Z$ of the virtually planned dental implants}

NOTE: Use the correct dental implant diameter and height (including cover screw) during the preoperative planning for correct comparison.

1. Click Construct | Point | Point and create a virtual point by using Ctrl + left click in the middle and top of the cover screws of the dental implants in the Mandible Pre-op file.

2. Click Construct | Point | Surface Point and create a virtual point by using Ctrl + left click in the middle and top of the cover screws of the dental implants in the Mandible Post-op file.

3. Right mouse button click on first dental implant in the Mandible Pre-op. Click Measuring Principle | Link to Actual Element. Select the same dental implant of the Mandible Post-op. Repeat this procedure for all dental implants.

4. Select all the points on the dental implants in both the Mandible Pre-op and the Mandible Post-op files in the left explorer menu. Click the magnifying glass on the toolbar above. A round toolbar pops up in the screen. Click Check and select $\mathbf{d X Y Z}$ to show the distance $\mathrm{XYZ}$ in mm per dental implant using the formula:

$d X Y Z=\sqrt{\left(\mathrm{x}^{2}-\mathrm{x}^{1}\right)^{2}+\left(\mathrm{y}^{2}-\mathrm{y}^{1}\right)^{2}+\left(\mathrm{z}^{2}-\mathrm{z}^{1}\right)^{2}}$

\section{Representative Results}

A James Brown class III mandibular defect was reconstructed at our department with the fibula free flap as a donor site. Direct guided dental implant placement was performed with the use of a fibula cutting guide which also included dental implant guides. The reconstruction was evaluated with the presented guideline. The coronal, axial and sagittal mandibular angle deviations $\left(^{\circ}\right)$ and six dental implant $X Y Z$ distances $(\mathrm{mm})$ were calculated and reported (Figure 4 and Figure 5).
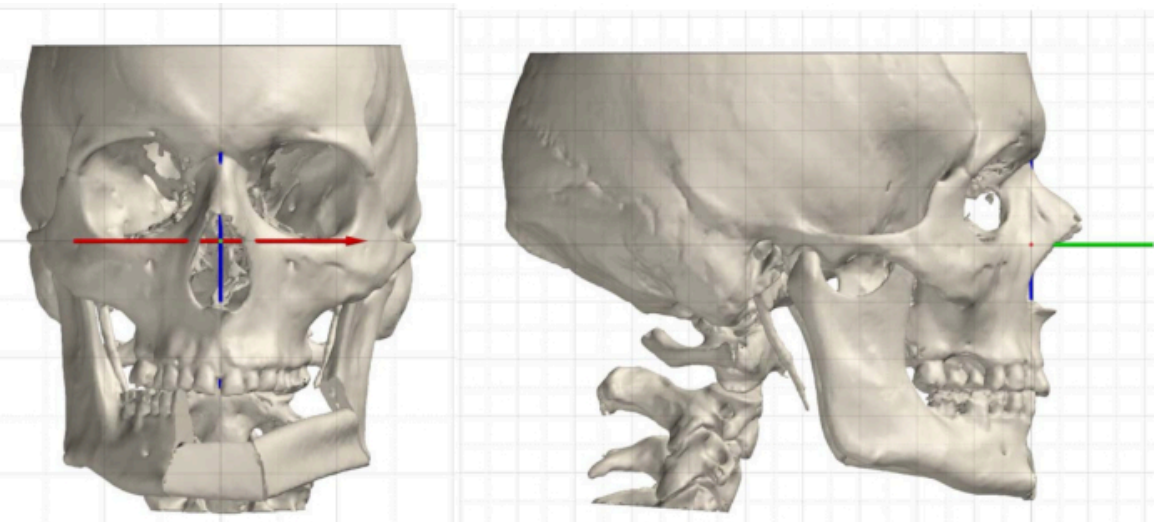

Figure 1: Uniform orientation of the preoperative STL model of the skull on the XYZ axis with the Frankfurt plane projected to the $Z$ axis (red line), the midsagittal plane projected to the $Y$ axis (green line), and the nasion projected to the $X$ axis (blue line). Please click here to view a larger version of this figure. 


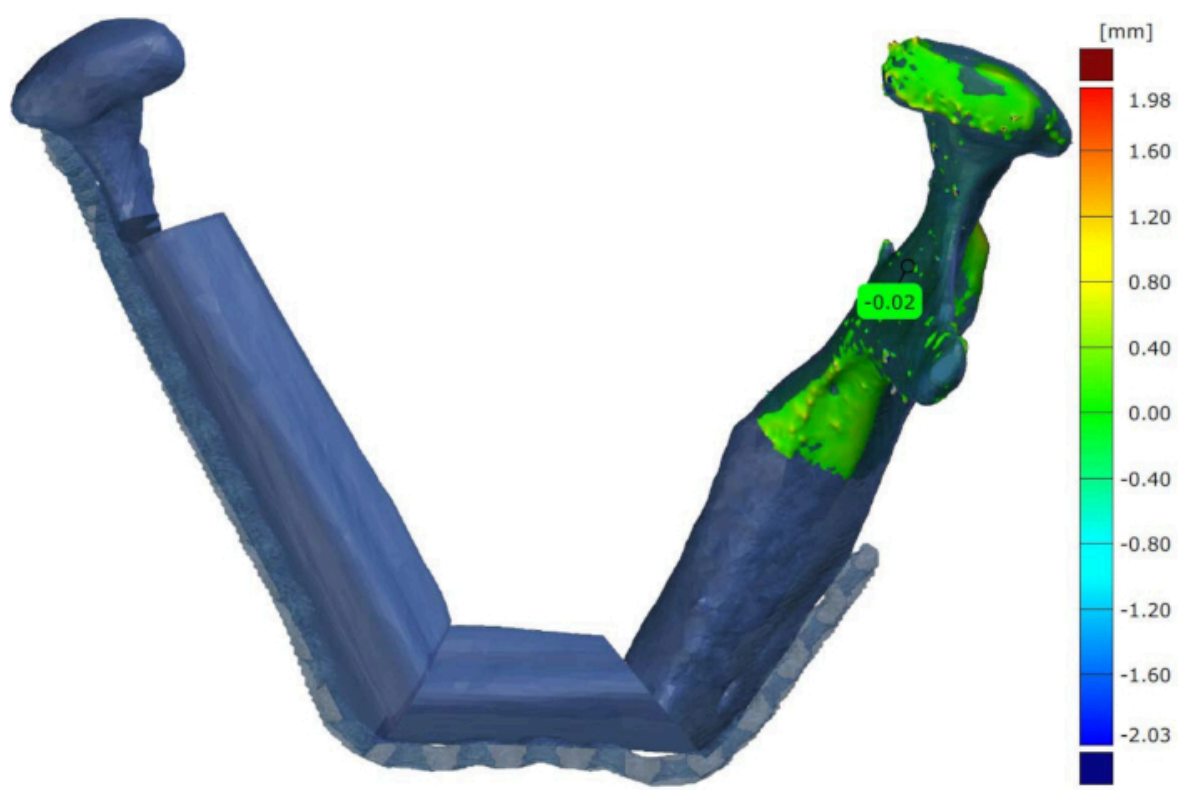

Figure 2: A part of the right side of the mandible (without involvement of osteosynthesis material which causes scattering) of the preoperative virtually planned STL model is superimposed on the postoperative STL model. Subsequently the CAS software is used to calculate the arithmetic mean. The $0.02 \mathrm{~mm}$ deviation between both volumes in this example falls within the norm $(<0.5 \mathrm{~mm})$ to proceed to the next step of the evaluation guideline. Please click here to view a larger version of this figure.

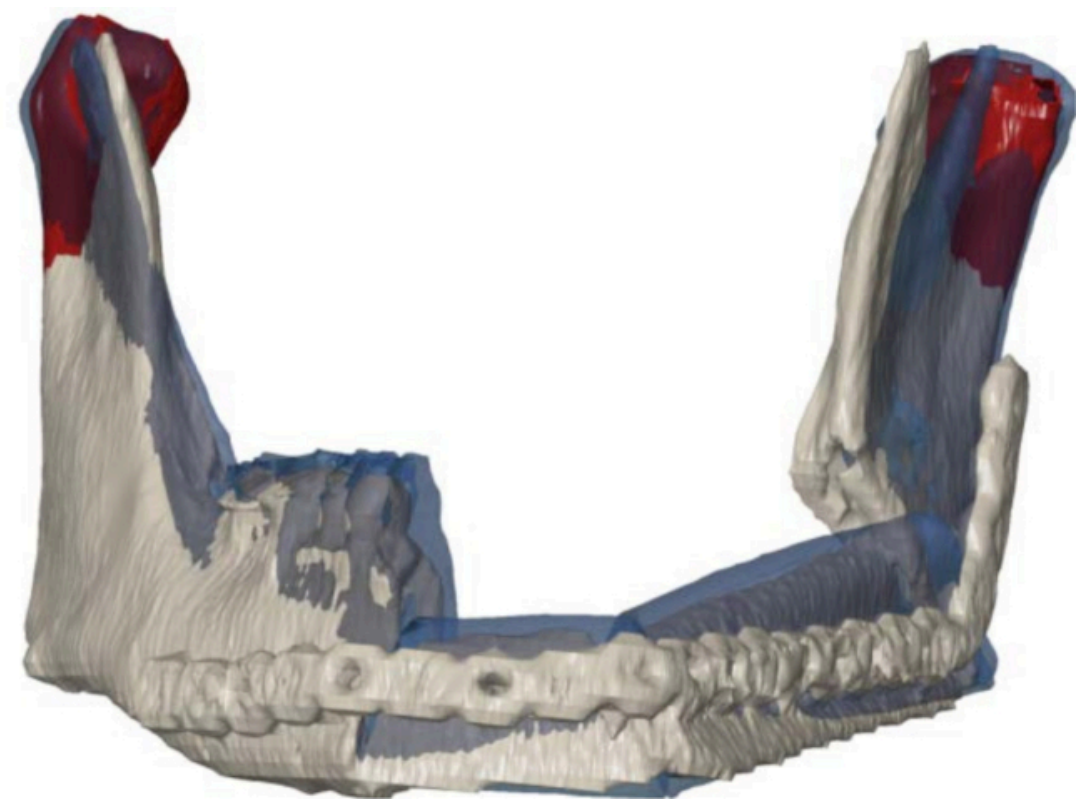

Figure 3: Superimposition of the postoperative STL model (grey) on the preoperative STL model, revised to the virtual plan (blue). Only both condylar processes are selected for the iterative closest-point algorithm (red). Please click here to view a larger version of this figure. 


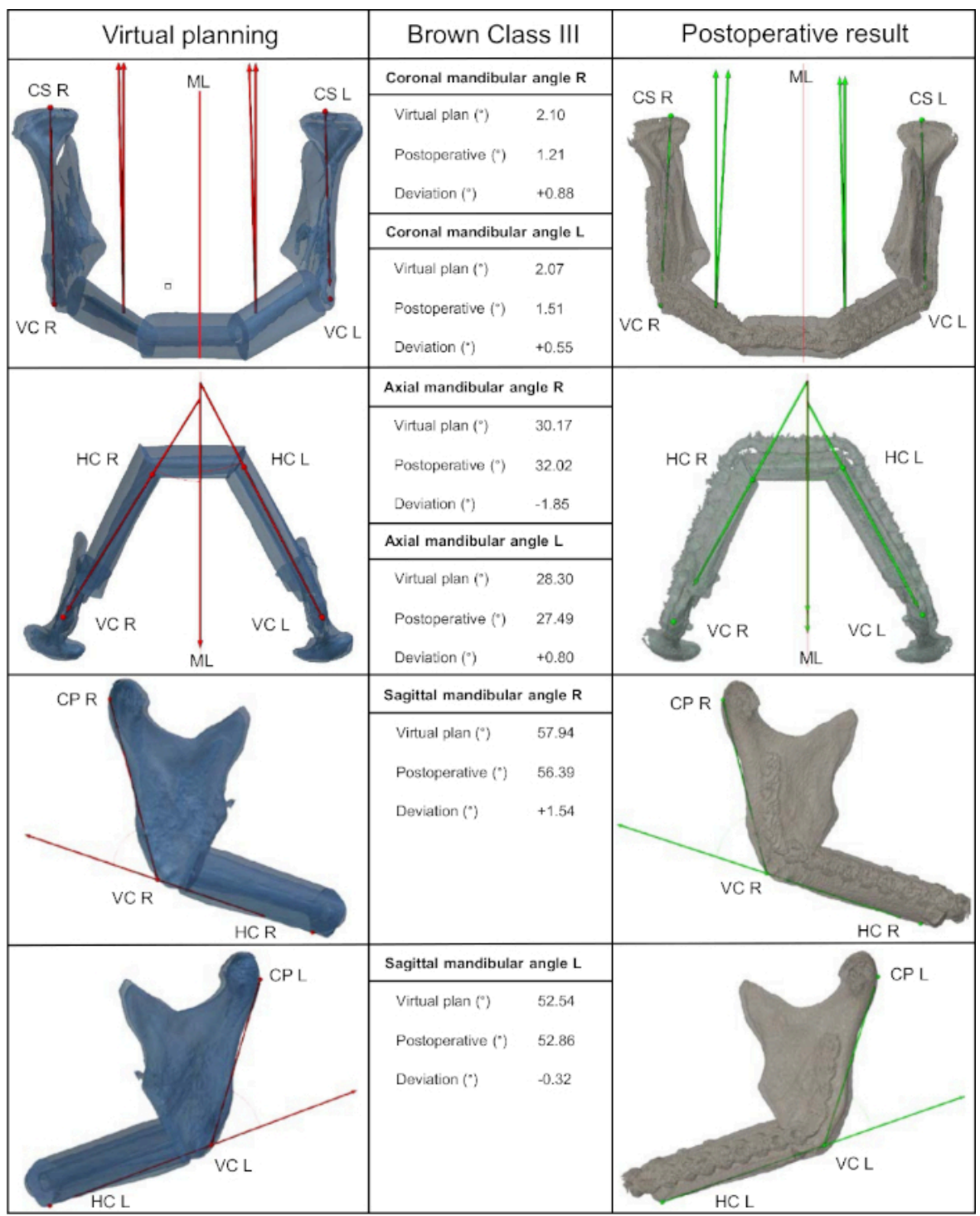

Figure 4: Reconstruction of a Brown class III defect using the fibula free flap as a donor site. In this example, six virtually planned dental implants are primary placed during the reconstruction using a 3D guide. The coronal, axial and sagittal angles are calculated on both the preoperative virtually planned 3D model and the postoperative 3D model. The deviations between the angles in degrees $\left({ }^{\circ}\right)$ are shown. CS, condyle superior; CP, condyle posterior; VC, vertical corner; HC, horizontal corner; ML, midsagittal line; FFF, fibula free flap. Please click here to view a larger version of this figure. 


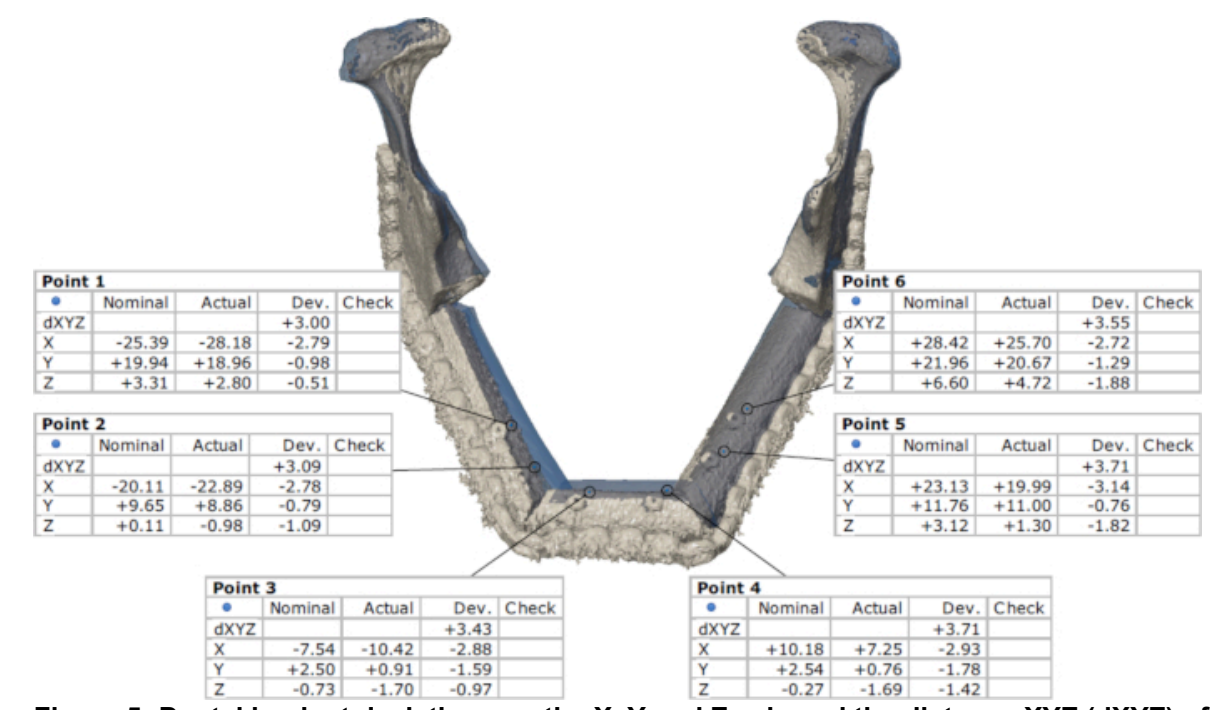

Figure 5: Dental implant deviations on the $X, Y$ and $Z$ axis and the distance $X Y Z(d X Y Z)$ of the six guided placed dental implants. Please click here to view a larger version of this figure.

Discussion

This postoperative evaluation guideline aims to facilitate increased uniformity of accuracy analysis of computer-assisted mandibular reconstructions. The focus is on four components determining the success of mandibular reconstruction: (1) the position of both condyles, (2) the angles of the osteotomy planes, (3) the size, position and fixation of the bone graft segments, and (4) the position of the guided dental implants (if immediate performed and included in the virtual planning).

In the first step of our proposed protocol, we recommend MDCT scanning for both pre- and postoperative imaging, because the quality of CT images affects the volume accuracy of segmented STL models. The largest volume deviations are found in STL models segmented out of cone beam computed tomography (CBCT) scanner DICOM data ${ }^{11}$. These volume deviations influence accuracy and fitting of 3D printed templates and guides, and thus also influences postoperative accuracy measurements between pre- and postoperative STL models. Therefore, we recommend the use of MDCT scanners in both pre- and postoperative imaging for mandibular reconstruction using CAS. Slice thickness is the most influencing factor in STL volume accuracy and should be set $<1.25 \mathrm{~mm}$. A higher slice thickness yields to loss of detail in the STL models and affects accuracy measurements ${ }^{12,13}$. A recently published systematic review on accuracy in mandibular reconstruction using CAS showed poor description in the materials and methods section of CT scanner parameters used by authors ${ }^{5}$. In our opinion, CAS studies should always specify the type and parameters of pre- and postoperative imaging modalities in the materials and methods section. In order to avoid long term changes in the volume, shape, and position of the segments of the bone graft, the postoperative MDCT scan should be performed within six weeks after reconstruction ${ }^{14}$. In case of adjuvant radiation therapy, use the first postoperative MDCT scan prior to the therapy to avoid radiation related pathology in the mandibular bone ${ }^{15}$.

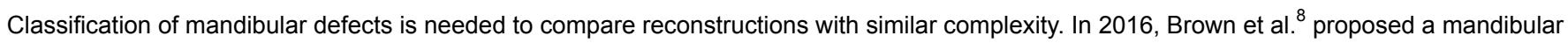
defect classification describing four classes, with a relationship between the class number and the complexity of the reconstruction. The alignment of pre- and postoperative STL models in the CAS software to evaluate the accuracy of the reconstruction introduces some difficulties. The superimposition software tool moves a selected part of an STL model (the source) to best match a fixed part of an STL model (the reference) using an iterative closest-point algorithm. However, superimposition of the entire (neo)mandible is inaccurate due to scattering of the reconstruction plate(s), which will lead to shifts of the entire reconstruction, not representing the postoperative clinical position of the mandible ${ }^{16}$. The same problem is introduced while superimposing isolated parts of the reconstruction ${ }^{17}$. Superimposition of the mandible including the maxilla and cranium is inaccurate because mouth opening will always be different during the pre- and postoperative scanning. Therefore, to evaluate the postoperative position of the (neo)mandible we decided to create mandibular angles (pioneered by De Maesschalck et al. ${ }^{18}$ ) on both pre-and postoperative STL models separately to bypass the superimposition problems. However, to evaluate the dental implant positions we necessarily needed to align both models, using the superimposition software tool. To align pre- and postoperative STL models with the closest approach to the clinical postoperative intermaxillary relation, we believe that superimposition of only both condylar processes is the most feasible, standardized and reproducible method. Although the postoperative position of both condyles can be affected by inaccurate neomandible reconstruction, the intermaxillary relation will accommodate to the midline and thus averages the position of both condyles around the midsagittal plane ${ }^{19}$. In our protocol, only the preoperative STL model is quickly fixed to the XYZ axis using a plane-line-point tool in the CAS software, representing a benchmark from which the postoperative deviations of the dental implants can be determined. The fixed skull position on the $\mathrm{XYZ}$ axis can lead to small cephalometric differences between cases. However, this has no influence on the dental implant measurements, because it has no consequences for the distance $X Y Z$ in mm between dental implant positions when the postoperative 3D model is superimposed onto the fixed preoperative $3 \mathrm{D}$ model with only both condyles selected for the iterative closest point algorithm.

As described above, De Maesschalck et al. ${ }^{18}$ pioneered an evaluation method for hard tissue accuracy of mandibular reconstruction using CAS, bypassing the need for osteotomy plane determination and bypassing the use of a superimposition tool. The most serious disadvantage of this method is that it failed to specify the method used to determine the midsagittal plane, which needs to be standardized and reproducible. Also, no virtually planned dental implants are included and a differentiation between complexity of mandibular reconstructions is lacking. We included the evaluation of postoperative positions of virtually planned dental implants in our protocol because the number of authors applying guided dental implants in the future is likely to increase. In 2016 , Schepers et al. ${ }^{20}$ proposed an excellent postoperative evaluation method for virtually 
planned dental implants in mandibular reconstruction using CAS by measuring the center point deviation $(\mathrm{mm})$ and angular deviation $\left({ }^{\circ}\right)$ per dental implant. The main limitation of this method is the quantity of measurements per implant which decreases the feasibility and results in loss of overview of accuracy of the entire reconstruction. We propose a more simplified method by determining one recapitulatory number per dental implant by measuring the distance $X Y Z$ ( $d X Y Z$ in $\mathrm{mm}$ ). With regard to dental rehabilitation, the position of the neck of the dental implant is decisive for future prosthetics. Therefore, our evaluation protocol recommends creating virtual points on the neck of the dental implants in the pre- and postoperative STL models. To keep the evaluation of the dental implants feasible we decided to skip angular deviation measurements, because angular deviations up to $15^{\circ}$ can be corrected with angled implant abutments.

Our proposed guideline is applicable for all types of donor sites and allows for different bone graft fixation possibilities. Also, CT scattering of metal fixation parts in the postoperative imaging will not influence measurements of the guideline ${ }^{5}$. In this evaluation guideline, we used Mimics inPrint 3.0 and GOM Inspect Professional 2019. However, the protocol describes software tools which are available in all CAS software packages. This guideline aims to contribute to a much more standardized and uniform approach to objectify relationships between accuracy and all different approaches during the CAS phases. There is abundant room for further progress in determining acceptable mandibular angle deviations per Brown class, their relationship with the postoperative positions of virtually planned dental implants, and acceptable dental implant deviations ( $d X Y Z)$ for future prosthetics. Currently, our department is conducting a multicenter study to validate this guideline in a large cohort, which also takes all the above-mentioned variables into account.

\section{Disclosures}

The authors have nothing to disclose.

\section{Acknowledgments}

This research did not receive any specific grant from funding agencies in the public, commercial, or not-for-profit sectors.

\section{References}

1. Rodby, K. A. et al. Advances in oncologic head and neck reconstruction: systematic review and future considerations of virtual surgical planning and computer aided design/computer aided modeling. Journal of Plastic, Reconstructive \& Aesthetic Surgery. 67 (9), $1171-1185$ (2014).

2. Rengier, F. et al. 3D printing based on imaging data: review of medical applications. International Journal of Computer Assisted Radiology and Surgery. 5 (4), 335-341 (2010).

3. Marro, A., Bandukwala, T., Mak, W. Three-Dimensional Printing and Medical Imaging: A Review of the Methods and Applications. Current Problems in Diagnostic Radiology. 45 (1), 2-9 (2016).

4. Mitsouras, D. et al. Medical 3D Printing for the Radiologist. Radiographics. 35 (7), 1965-1988 (2015).

5. van Baar, G. J. C., Forouzanfar, T., Liberton, N., Winters, H. A. H., Leusink, F. K. J. Accuracy of computer-assisted surgery in mandibular reconstruction: A systematic review. Oral Oncology. 84, 52-60 (2018).

6. European Union Medical Device Regulation. Regulation (EU) 2017/745 of the European Parliament and of the Council of 5 April 2017 on medical devices, amending Directive 2001/83/EC, Regulation (EC) No 178/2002 and Regulation (EC) No 1223/2009 and repealing Council Directives 90/385/EEC and 93/42/EEC. Official Journal of the European Union. 60 (117), 1-332 (2017).

7. van Baar, G. J. C., Liberton, N., Forouzanfar, T., Winters, H. A. H., Leusink, F. K. J. Accuracy of computer-assisted surgery in mandibular reconstruction: A postoperative evaluation guideline. Oral Oncology. 88, 1-8 (2019).

8. Brown, J. S., Barry, C., Ho, M., Shaw, R. A new classification for mandibular defects after oncological resection. Lancet Oncology. 17 (1), e23-30 (2016).

9. Pittayapat, P. et al. Three-dimensional Frankfort horizontal plane for $3 \mathrm{D}$ cephalometry: a comparative assessment of conventional versus novel landmarks and horizontal planes. European Journal of Orthodontics. 40 (3), 239-248 (2018).

10. Green, M. N., Bloom, J. M., Kulbersh, R. A simple and accurate craniofacial midsagittal plane definition. American Journal of Orthodontics and Dentofacial Orthopedics. 152 (3), 355-363 (2017).

11. van Eijnatten, M. B. F., de Graaf, P., Koivisto, J., Forouzanfar, T., Wolff, J. Influence of ct parameters on stl model accuracy. Rapid Prototyping Journal. 24 (4), 679-685 (2017).

12. Whyms, B. J. et al. The effect of computed tomographic scanner parameters and 3-dimensional volume rendering techniques on the accuracy of linear, angular, and volumetric measurements of the mandible. Oral Surgery, Oral Medicine, Oral Pathology, and Oral Radiology. 115 (5), 682-691 (2013).

13. Taft, R. M., Kondor, S., Grant, G. T. Accuracy of rapid prototype models for head and neck reconstruction. Journal of Prosthetic Dentistry. 106 (6), 399-408 (2011).

14. Disa, J. J., Winters, R. M., Hidalgo, D. A. Long-term evaluation of bone mass in free fibula flap mandible reconstruction. The American Journal of Surgery. 174 (5), 503-506 (1997).

15. Jereczek-Fossa, B. A., Orecchia, R. Radiotherapy-induced mandibular bone complications. Cancer Treatments Reviews. 28 (1), 65-74 (2002).

16. Tarsitano, A. et al. Accuracy of CAD/CAM mandibular reconstruction: A three-dimensional, fully virtual outcome evaluation method. Journal of Cranio-Maxillofacial Surgery. 46 (7), 1121-1125 (2018).

17. Roser, S. M. et al. The accuracy of virtual surgical planning in free fibula mandibular reconstruction: comparison of planned and final results. Journal of Oral and Maxillofacial Surgery. 68 (11), 2824-2832 (2010).

18. De Maesschalck, T., Courvoisier, D. S., Scolozzi, P. Computer-assisted versus traditional freehand technique in fibular free flap mandibular reconstruction: a morphological comparative study. European Archives of Oto-Rhino-Laryngology. 274 (1), 517-526 (2017).

19. Hidalgo, D. A., Pusic, A. L. Free-flap mandibular reconstruction: a 10-year follow-up study. Plastic and Reconstructive Surgery. 110 (2), 438-449; discussion 450-451 (2002). 
20. Schepers, R. H. et al. Accuracy of secondary maxillofacial reconstruction with prefabricated fibula grafts using 3D planning and guided reconstruction. Journal of Cranio-Maxillofacial Surgery. 44 (4), 392-399 (2016). 